Zur Zusammenarbeit von Verbraucherorganisationen mit Unternehmen

\section{Mesalliance unter Ungleichen}

Auch Verbraucherorganisationen arbeiten mit Unternehmen zusammen. Sie partizipieren bei umweltfreundlicher oder ethisch motivierter Produktentwicklung und versuchen vor der Vermarktung neuer Produkte Konsumenteninteressen durch verschiedene Dialogformen zu wahren. Das Verhältnis zwischen Firmen und Verbraucherschützern ist jedoch nicht unstrittig. Besonders die zunehmende finanzielle Unterstützung von Verbraucherorganisationen durch Firmen als Folge der staatlichen Förderungskürzung birgt weiteren Konfliktstoff.

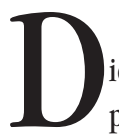

Von Volkmar Lübke ie häufigsten Ursachen für Verbraucherprobleme und -beschwerden sind Konflikte aufgrund des Verhaltens der Anbieter von Waren und Dienstleistungen. Verbraucherorganisationen positionieren sich in diesen Fällen entsprechend ihrer Rolle als Interessenvertreterinnen der betroffenen Verbraucher grundsätzlich konträr zu den beteiligten Anbietern. Doch haben es sich Verbraucherorganisationen längst abgewöhnt, das Verhältnis zur Anbieterseite nur als ,,kämpferisches Gegeneinander" zu definieren. Die Palette der Arbeitsinstrumente, die zur Minderung der Unterlegenheit von Verbrauchern im Marktgeschehen eingesetzt werden können, reicht heute von Musterprozessen über Abmahnverfahren, vergleichende Produktund Unternehmensbewertungen, Gegeninformationen und Dialogen bis zu den unterschiedlichsten Kooperationsformen mit Anbietern.

\section{- Ende des kalten Krieges}

Im Rahmen der Karrieren von Leitbildern wie dem ökologischen oder nachhaltigen Konsum hat sich bei Verbraucherschützern besonders eine Erkenntnis durchgesetzt: Es reicht nicht aus, mit kritischen Interventionen gegen problematisches Anbieterverhalten vorzugehen, wenn nicht gleichzeitig Marktpotenziale für positive Alternativen entwickelt und gefördert werden. Der damalige Bundesvorsitzende der von Mitgliedern getragenen Verbraucher Initiative, Gerd Billen, verkïndete im Jahr 1991 programmatisch: „Die Zeit des kalten Krieges zwischen Bürgerinitiativen und Verbrauchergruppen auf der einen, den Unternehmen auf der anderen Seite ist vorbei“. In der Folge wurden auch Projekte durchgeführt, die dieser neuen Linie entsprachen, allerdings zum Teil auch Konflikte mit Mitgliedern auslösten und sogar zu einzelnen Austritten aus dem Verband führten. So entsprach zum Beispiel die Unterstïtzung des Projektes „TransFair-Kaffee“ nicht den fundamentalistischen Einstellungen einiger entwicklungspolitischer Verbände.

Der damalige Dachverband der öffentlich finanzierten Verbraucher Zentralen (AGV) beschloss noch im Januar 1992 ein Grundsatzpapier, das sogar Dialoge mit der Anbieterseite als eine „Kooperationsform“ behandelte und davor warnte, dass die „öffentliche Glaubwürdigkeit der Verbraucherorganisationen untergraben" werden könnte, wenn Dialoge auf eine ,unternehmensorientierte Steuerung verbraucherpolitischer Konflikte hinauslaufen“ sollten. In der Zwischenzeit ist es allerdings auch bei Verbraucher Zentralen zu den unterschiedlichsten Kooperationsformen gekommen - vor allem mit Unternehmen, in denen der Staat noch Eigentumsanteile hat.

Das Hauptrisiko für Verbraucherorganisationen, die mit Anbietern kooperieren besteht in einem möglichen Verlust ihrer Unabhängigkeit, faktisch oder auch ,nur“ in der öffentlichen Wahrnehmung, und damit ihrer Glaubwürdigkeit - die ihr wichtigstes ,Kapital“ darstellt. Darüber hinaus ist ein relevantes ,,juristisches Restrisiko“ nicht zu übersehen. So könnte sich bei einem Konflikt mit einem Anbieter aus der gleichen Branche wie der, mit dem kooperiert wird, die Tatsache der Zusammenarbeit mit einem Wettbewerber als juristisch nachteilig herausstellen.

Neben dem oben beschriebenen und verbraucherpolitisch gut begründbaren Paradigmenwechsel beim Umgang mit der Anbieterseite gibt es auch Wirkungszusammenhänge, die im Hinblick auf die Zukunft der Verbraucherarbeit bedenklicher stimmen. In den letzten Jahrzehnten hat die Mittelknappheit der öffentlichen Hand und der Rückzug des Bundes aus der Finanzierung der Verbraucherarbeit der Länder zu existenzbedrohenden Einnah- merückgängen der staatlich finanzierten Verbraucher Zentralen in den Bundesländern geführt. Insbesondere in den letzten drei Jahren häuften sich dort deshalb Schließungen von Beratungsstellen und Entlassungen von Mitarbeitern. Die Einnahmeverluste haben die meisten Verbraucher Zentralen trotz aller Anstrengungen nicht wettmachen können. In dieser ökonomischen Zwangslage werden Kooperationen mit der Anbieterseite zunehmend auch als Möglichkeit zur Entlastung der angespannten Haushaltssituation gesehen.

\section{Unternehmenszuwendungen steigen}

Bereits im Jahr 1994 ging die Verbraucher-Zentrale Hamburg als erste dazu über, als Finanzierungsquelle auch Zuwendungen von Unternehmen zu akzeptieren. Dies geschah allerdings unter drei Voraussetzungen: Bedingungsfreiheit und insbesondere keine Akzeptanz von Sponsoring, keine Werbung mit der Spende durch die Unternehmen sowie Transparenz in Form von rückhaltloser Veröffentlichung im eigenen Jahresbericht. Inzwischen betreibt die Verbraucher-Zentrale Hamburg die Akquisition zusätzlicher Mittel über einen eigenen Förderverein sehr erfolgreich. Der Jahresbericht für das Jahr 1994 wies erstmals freiwillige Beiträge von Unternehmen der anbietenden Wirtschaft nach, die insgesamt circa einem Prozent der Eigeneinnahmen ausmachten. Für das Jahr 1998 wurde der entsprechende Anteil bereits mit fünf Prozent beziffert. Auch Verbraucher Zentralen anderer Bundesländer sehen inzwischen in der Akquisition von Mitteln der Anbieterseite eine letzte Möglichkeit, ihre Einnahmesituation zu verbessern. Es ist unübersehbar, dass viele Bundesländer sich aus der öffentlichen Finanzierung der Verbraucherarbeit auf der Basis des Subsidiaritätsprinzips Schritt für Schritt herausziehen möchten. Ob das Verhältnis zwischen Verbraucher Zentralen und Unternehmen in dieser Situation weiterhin als die erwünschte ,symmetrische Beziehung“ unter gleich starken und konfliktfähigen Marktpartnern entwickelt werden kann, muss bezweifelt werden.

\section{Der Autor}

Volkmar Lübke ist Vorstandsmitglied der Verbraucher Initiative Berlin und seit 1980 in und für Verbraucherorganisationen tätig.

Kontakt: Jägerndorfer Zeile 3, 12205 Berlin.

Tel. und Fax 030-80581318,

E-Mail: v.luebke@gmx.de 
(c) 20I0 Authors; licensee IÖW and oekom verlag. This is an article distributed under the terms of the Creative Commons Attribution Non-Commercial No Derivates License (http://creativecommons.org/licenses/by-nc-nd/3.o/), which permits unrestricted use, distribution, and reproduction in any medium, provided the original work is properly cited. 